\title{
Resilience Status on Young Athletic Athletes in Response to Covid-19 Pandemic
}

\author{
$1^{\text {st }}$ Adiska Rani Ditya Candra ${ }^{1}, 2^{\text {nd }} \operatorname{Hermahayu}^{2}$ \\ \{adiska_rani@mail.unnes.ac.id ${ }^{1}$, hermahayu@ummgl.ac.id ${ }^{2}$ \} \\ Universitas Negeri Semarang, Semarang, Indonesia ${ }^{1}$ \\ Universitas Muhammadiyah Magelang, Magelang, Indonesia ${ }^{2}$
}

\begin{abstract}
This study aims to examine the resilience status of Junior Athletic Athletes during Covid-19 Pandemic. The drastic changes have an impact on the growth and development of sports achievement. The program is still running with the Covid-19 Pandemic, but there are alot of restrictions which cause concern for athletes and the emergence of performance problems. The results of this study indicate that Long-Term Sports Center Program (PSOJP) athletic athletes have an excellent resilience status of $20 \%, 20 \%$ have a fairy good category and $60 \%$ are in a very good category. The recommendations of this study are aimed at coaches, even though the athletes have a fairly good resilience status, there is a need for assistance and strategies for athletes to be able to increase psychological resilience and be ready to survive in difficult conditions.
\end{abstract}

Keywords: Resilience status, young athletes, long-term athlete, covid-19

\section{Introduction}

Resilience is a combination or combination of several contributing psychological factors. Many studies have explained the main factors in influencing the contribution and develope of resilience in a person. Resilience can be defined as a process of adaptation to someone from adversity, trauma, tragedy, threats or even things that become significant sources of stress. For example, family problems, elationship problems, serious keshetan problems, financial stress, situations that are difficult to accept and many others. In other words, resilience is bouncing back from a difficult situation [1].

Resilience is defined as a process that is based on the positive role of differences in individual responses to stress and difficulties so as to enable the individual to become resilient $[2,3]$. The same thing was conveyed in previous studies, namely resilience refers to the identification of characteristics in an individual that allows him to be resilient [4]. Closely related to sports, especially competitive sports, many things can happen and make these athletes experience difficulties, stress and even tend not to survive. Especially for student or student athletes, some are able to survive but others may not.

Several things are related to high training load and academic studies such as, selection of exercise schedules, the selection process, biological changes during puberty, personal conflicts, and stress from the sports environment that can potentially cause stress [5]. During the training process, athletes are also vulnerable to various difficult situations that cause stress, athletes may experience injuries, training loads that are too heavy so they experience fatigue, which of course will cause a decrease in sports performance. The demand for winning a competition also triggers stress, which means that athletes must be able to adapt 
positively to survive to grow into a ready and resilient person [6-8] Attempts and successes in achieving resilience have been noted in previous studies that traits of a sports environment such as interpersonal relationships, and positive coach behavior supporting gymnasts through challenges can encourage athletes to overcome failure. The results of this study state that gymnastics athletes can develop resilience as well as skills, self-efficacy, and self-esteem through positive behaviors given by the coach and are considered the right path for future coaching practices [9].

The pandemic crisis initially affected various sectors of life, especially the health sector, on the other hand, there are the economic, education, industrial, tourism, social, employment, and of course sports sectors [10-12]. More clearly, Paoli explained a very clear impact on the sports sector, namely the application of motion restrictions (lockdown) to make athletes normally stop their training activities and reduce the volume and intensity of training for several periods, and result in the closure of sports venues and athletes [13]. These situations are what make the athlete experience very drastic changes in the training period according to the race and match schedule, a decrease in performance due to a decrease in training intensity and volume. However, on the other hand, athletes must have targets and achievements and the status of increasing achievement during the coaching process so that it causes athletes to experience stress and emotional instability, including junior athletes in the Long-Term Sports Centralization Program (PSOJP) Jawa Tengah.

Based on the background above, conditions that tend to make athletes experience stress must get attention and this situation is considered as adversity that can interfere with the mental condition of athletes to be able to survive and maintain their psychological condition during the Covid-19 pandemic. In line with this, the research is to measure and determine the resilience status of Athletic PSOJP Athletes in response to Covid-19.

\section{Methods}

This study aims to measure the extent of the resilience status of the athletic PSOJP athletes during the Covid-19 pandemic. Participants in this study involved athletes from Central Java PSOJP Athletics. Participants consisted of 10 athletes with a distribution of 6 male athletes and 4 female athletes. Athletes aged 14 to 20 years $(\mathrm{M}=18.00, \mathrm{SD}=2,44)$. Participants who became respondents in this study were active athletes who underwent a training process during the Covid-19 pandemic with strict health protocols. The sample used in this study used a total sampling of all PSOJP Athletic Athletes.

The procedure in this study is to follow the covid-19 protocol guidelines by not being directly involved with athletes. The measurement process uses a questionnaire that has been made through the online questionnaire media platform.

The instrument used in this study was The Brief Resilience Scale (BRS) instrument developed by Smith et al [14]. The BRS questionnaire includes 6 statements indicating the psychological resilience of athletes in facing the situation during the Covid pandemic. Apart from that, to trace further the researchers also looked at it by conducting specific interviews. The measurement scale uses a Likert scale with a scale point of $1=$ Strongly Disagree, $2=$ Disagree, 3 = neutral, $4=$ Agree, 5 = strongly agree.

The calculation of the percentage results will provide the conclusions presented in the percentage classification as follows : 
Table 1. Percentage Classification

\begin{tabular}{cc}
\hline Presentase & Klasifikasi \\
\hline $80-100 \%$ & Excelent \\
$60-79,99 \%$ & Very Good \\
$40-59,99 \%$ & Good \\
$20-39,99 \%$ & Fair \\
$0-19,99 \%$ & Poor \\
\hline
\end{tabular}

The distributions of Resileince statemen are avalaible in this table bellow:

Tabel 2. The Brief Resilience Scale: Items and Factor Loadings

\begin{tabular}{ccl}
\hline No & Indicator (BRS) & Item Scale \\
\hline 1 & I tend to bounce back quickly after hard times \\
2 & I have a hard time making it trogh streesful event (R) \\
3 & Resilience & It doesnt not take me long to recover from a stressfull event \\
4 & It is hard for e to snap back when something bad happens (R) \\
5 & I usually come through difficult timess with little trouble \\
6 & T tentd to take a long itme to get over set-backs in my life (R) \\
\hline
\end{tabular}

\section{Result and Discussion}

\subsection{Results}

The results of the descriptive analysis will be described in the table below based on $\mathrm{N}$ (total of respondents). The results of the descriptive analysis will be shown in table 1 while the percentage results are presented in table no.2. The descriptive results that have been obtained are:

Tabel 3. Tabel Analisis Deskriptif

\begin{tabular}{cccccccc}
\hline \multicolumn{7}{c}{ Descriptive Statistics } \\
\hline Statement & N & Range & Minimum & Maximum & Mean & Std. Deviation & Variance \\
\hline 1 & 10 & 2 & 3 & 5 & 4.60 & .699 & .489 \\
2 & 10 & 3 & 2 & 5 & 3.80 & 1.229 & 1.511 \\
3 & 10 & 3 & 2 & 5 & 4.10 & 1.101 & 1.211 \\
4 & 10 & 3 & 2 & 5 & 3.50 & 1.269 & 1.611 \\
5 & 10 & 2 & 2 & 4 & 3.60 & .699 & .489 \\
6 & 10 & 4 & 1 & 5 & 3.60 & 1.075 & 1.156 \\
$\begin{array}{c}\text { Valid N } \\
\text { (listwise) }\end{array}$ & 10 & & & & & & \\
\hline
\end{tabular}


Based on table no. 3 can be inferred that the lowest score of the respondents, total of 10 athletes, with the lowest score of 1 and the highest is 5 . The lowest score from the Mean data obtained is 3,50 in a statement number 4 while the highest score from the mean data above is of 4.60 from the statement nuber 1 . Below is the descriptive result of the percentage.

Tabel 4. Descriptive Percentage of Resilience of PSOJP Athletic Athletes Jawa Tengah

\begin{tabular}{ccc}
\hline Percentage Inyterval & Criteria & Percentage \\
\hline $80 \%-100 \%$ & Excelent & $20 \%$ \\
$60 \%-79,99 \%$ & Very Good & $60 \%$ \\
$40 \%-59,99 \%$ & Good & $20 \%$ \\
$20 \%-39,99 \%$ & Fair & $0 \%$ \\
$0 \%-19,99 \%$ & Poor & $0 \%$ \\
\hline
\end{tabular}

Based on the data shown in table 2 it can be seen that the Central Java PSOJP Athletic athletes are divided into 3 criteria, namely the category Good, Very Good, Excellent. The results of the percentage obtained indicate that athletes who have an Excellent level of resilience are still far from half. It can be seen that only $20 \%$ of athletes have an excellent level of resilience. Furthermore, $60 \%$ of the total number entered into the very good level, and $20 \%$ of athletes were categorized as having a fairly good resilience.

In addition to percentage data, resilience measurements are also taken from the athlete's point of view in solving psychological problems during training, especially during the Covid pandemic. The point of view of the athlete is taken from the assessment of (1) Movement Restriction, (2) Action as a positive response. Several athletes conveyed what point of view they felt in an open questionnaire such as:

I am very disappointed and saddened by this situation, especially when there is a limitation on space for movement (Respondent 4). my practice was interrupted (Respondent $3)$. Very disappointed because we let go of the target and the training was not optimal (Respondent 8).

And in the form of treatment to overcome negative thoughts to stay positive, some of them are by watching movies, looking for entertainment, playing cellphones, worshiping and praying, and staying enthusiastic even though you do not do routine exercise activities. They try to keep their spirits up in any condition because maybe they think this is what can be done.

\subsection{Discussions}

The purpose of this study was to measure the level of resilience of Athletic PSOJP Athletes in response to the Covid-19 pandemic which has an impact on athlete's training and several other aspects. From the data analysis that has been described, the results show that PSOJP Athletic athletes who have excellent psychological resilience are only $20 \%$ and the others are in the good category of $60 \%$ and $20 \%$ are in the good enough category. This proves that the psychological resilience status of the Athletic PSOJP Athletes is in a good category or even tends to be in the middle of not reaching a very good category. This is of course a question why some athletes do not get the maximum criteria for measuring psychological resilience. 
Resilience can be influenced by several factors. According to Fletcher, the resilience of athletes is influenced by the stressors they face. And in the resilience formation process, there are 5 main supporting factors, namely motivation, focus, perceived social support, confidence, and positive personality [4]. These factors are the main capital informing the resilience of athletes to be able to survive and maintain their psychological condition to remain at the highest position. From the athlete's point of view, it is clear that most athletes have a very large sense of disappointment and sadness due to the condition of the athletes who have to stay at home and also target sports events that are targeted must retreat and even disappear and in building their physical performance they must start from scratch. Besides, the athlete's concern will lose to other athletes if they have less preparation. Some of these things are also taken into account by the stressor level of the athlete.

Every athlete has different sources of stress and their way of dealing with or dealing with these stressors will be different [15]. In the case of PSOJP athletes, their athletics is one of the sports that needs outdoor training because it is related to that during the Covid-19 pandemic, athletes did not have the intensity of outdoor training because of safety that must be maintained. Besides, PSOJP athletes are young athletes, most of whom still have student status and students who are required to have to work extra hard in learning through an online system that they have never done before. These various sources of stress make athletes more likely not to be able to survive the conditions that force them to do so.

Gould et al. Also conveyed that individuals and institutions influence psychological development for the community, family, and also the individual itself, including people who are not related to sports, the sports environment, and the process of exercising. This is in line with the growth and development of resilience levels which are not only developed cognitively but also from the family, community, and all sports processes and the environment around the individual (athlete) themself [16].

The results of the medium resilience status of athletic PSOJP athletes provide recommendations and information for athletes that the athlete conditions is not good enough. This should be a consideration for the coach in developing a mental resilience and training program for the athlete. Moreover, strategies are given to athletes in the recovery of psychological conditions [17]. Such as coping strategies in solving problems and situations that threaten athletes and in this study are related to the recovery of feelings of disappointment, and increase in self-confidence and motivation and positive thinking that achievement can still be pursued with serious training and still adhere to health protocols. Another strategy used by athletes and coaches is to redefine the main objectives in achieving the following achievements with goals in the short, medium, and long term. This is needed to foster enthusiasm, confidence, and optimism in athletes related to responsibility and discipline to carry out the process of achieving targets with maximum effort

\section{Conclussions}

The purpose of this study was to measure the resilience status of Central Java Athletic Psojp athletes in response to covid-19. In the research process, things that become the main focus are the level of resilience and also the point of view related to restriction of motion and actions taken by athletes in the recovery phase or solving sports training problems. The results of this study indicate that PSOJP Athletic athletes have a good resilience status even though some of them as much as $20 \%$ have a moderate level of resilience. Based on these results, the trainer should pay attention to what factors can influence this condition. The coach's consideration of the psychological/mental condition in sports must be paid more 
attention to be able to restore the condition and restore the athlete to stay focused, confidently, and build his self-confidence again. Athletes who succeed at the level of resilience are considered to have been able to overcome the problems they experience and be able to withstand difficult conditions.

Some of the actions that can be taken by the coach in restoring the condition and increasing the level of athlete's resilience are psychological skills training which includes motivation, self-confidence focus, and self-determination, then the implementation of goal setting as a coping strategy for athletes. And further research can be investigated further about how factors can influence or specifically influence psychological resilience in athletes.

\section{References}

[1.] Association AP. THe Road To Resilience.

[2.] Flach F. Resilience: Discovering a new strength at times of stress. Resilience: Discovering a new strength at times of stress. New York, NY, US: Ballantine Books; 1988. xv, 270-xv, 270.

[3.] Rutter M. Resilience in the face of adversity. Wool Rec [Internet]. 2001 Dec 29 [cited 2021 Mar 28];160(3686):31. Available from: https://www.cambridge.org/core/product/identifier/S0007125000208775/type/journal_ article

[4.] Fletcher D, Sarkar M. A grounded theory of psychological resilience in Olympic champions. Psychol Sport Exerc [Internet]. 2012;13(5):669-78. Available from: http://dx.doi.org/10.1016/j.psychsport.2012.04.007

[5.] Caine D, Walch T, Sabato T. The elite young athlete: strategies to ensure physical and emotional health. Open Access J Sport Med. 2016; Volume 7:99-113.

[6.] Tabei Y, Fletcher D, Goodger K. The relationship between organizational stressors and athlete burnout in soccer players. J Clin Sport Psychol. 2012;6(2):146-65.

[7.] Moen F, Federici RA, Abrahamsen F. Examining possible Relationships between mindfulness, stress, school- and sport performances and athlete burnout. Int J Coach Sci. 2015;9(1):3-19.

[8.] De Francisco C, Arce C, Vílchez M del P, Vales Á. Antecedents and consequences of burnout in athletes: Perceived stress and depression. Int $\mathrm{J}$ Clin Heal Psychol. 2016;16(3):239-46.

[9.] White RL, Bennie A. Resilience in youth sport: A qualitative investigation of gymnastics coach and athlete perceptions. Int J Sport Sci Coach. 2015;10(2-3):37993.

[10.] Settersten RA, Bernardi L, Härkönen J, Antonucci TC, Dykstra PA, Heckhausen J, et al. Understanding the effects of Covid-19 through a life course lens. Adv Life Course Res. 2020;45(July):100360.

[11.] Haleem A, Javaid M. Effects of COVID-19 pandemic in daily life [Internet]. Elsevier; 2020. Available from: www.elsevier.com/locate/cmrp

[12.] Akbulaev N, Aliyev V. The effect of coronavirus sars-cov-2 in the tourism industry in Africa. J Environ Manag Tour. 2020;11(8):1938-47.

[13.] Paoli A, Musumeci G. Elite athletes and COVID-19 lockdown: Future health concerns for an entire sector. J Funct Morphol Kinesiol. 2020;5(2):10-2.

[14.] Smith BW, Dalen J, Wiggins K, Tooley E, Christopher P, Bernard J. The brief resilience scale: Assessing the ability to bounce back. Int $\mathrm{J}$ Behav Med. 2008;15(3):194-200. 
[15.] Khoirunnisa ' Miftakhul Jannah. RESILIENSI PADA ATLET RENANG. J Character. 2014;3(2):1-7.

[16.] Gould D, Dieffenbach K, Moffett A. Psychological characteristics and their development in Olympic champions. J Appl Sport Psychol. 2002;14(3):172-204.

[17.] Secades XG, Molinero O, Salguero A, Barquín RR, de la Vega R, Márquez S. Relationship between resilience and coping strategies in competitive sport. Percept Mot Skills. 2016;122(1):336-49. 